\title{
Continuous time system identification using subspace methods
}

\author{
Rosmiwati Mohd-Mokhtar* Liuping Wang*
}

(Received 7 November 2005, revised 15 April 2007)

\begin{abstract}
System identification is a well known technique for developing mathematical models based on plant input and output data sequences. Models that describe the systems may be in various forms and one of the possibilities is a state space model formulation. The state space mathematical modelling involves vectors and matrices in a unique geometrical framework. It offers the key advantages on providing low parameter sensitivity with respect to perturbation for high order systems and also has shown its ability to present multi-input and multioutput systems with minimal state dimensions. We use a time domain subspace approach in conjunction with Laguerre filters and instrumental variables to develop a mathematical formulation of the state space model for identification of a continuous time system. The method aims
\end{abstract}

*School of Electrical \& Computer Engineering, RMIT University, Melbourne, Australia. mailto:rosmiwati@ieee.org,

mailto:rosmiwati.mohdmokhtar@student.rmit. edu . au

See http://anziamj.austms.org.au/V47EMAC2005/Mohd for this article, (C) Austral. Mathematical Soc. 2007. Published June 26, 2007. ISSN 1446-8735 
at searching for accurate matrices of the state space model to ensure that the constructed model closely mimics the actual system as well as provide information for the purpose of control system design. The subspace identification algorithm provides state space models with better conditioning, improved quality and easily maintainable parametrisation. The algorithm is validated with identification of two systems: a simulated plant, and a magnetic bearing system. For both systems, the computer simulation results demonstrate that the obtained model describes the system closely.

\section{Contents}

1 Introduction

2 Continuous time system identification

2.1 Constructing filtered data matrices . . . . . . . . . . . C719

2.2 Identification using a causal IV . . . . . . . . . . . . C C721

2.3 Identification algorithm . . . . . . . . . . . . . . C722

3 Simulation examples $\mathbf{C 7 2 3}$

3.1 Coloured noise . . . . . . . . . . . . . . . . C723

3.2 Magnetic bearing system . . . . . . . . . . . . C725

4 Conclusion

References

C729

\section{Introduction}

System identification provides a useful means to obtain mathematical models for controller design $[1,2]$. The identified models predict dynamic proper- 
ties of a given system under various operating conditions. Nowadays, system identification is considered as a well known technique for developing mathematical models based on plant input and output data sequences. The motivation and goal of researching into this area partially lie on achieving an accurate plant model which therefore will be useful for further investigation of the system controller design. This article studies the continuous time system, and the model is constructed in the framework of state space model formulation. The state space mathematical realisation involves vectors and matrices in a unique geometrical framework. It offers the key advantages of providing low parameter sensitivity with respect to perturbation for higher order systems and also has shown its ability to present multi-input and multioutput systems with minimal state dimensions.

A time domain subspace approach builds the state space model. Since the first introduction, subspace methods have shown promising achievement in developing a model for application such as flexible structure [3, 4], flexible aircraft [5], aircraft dynamics [6], power transformer [7], antenna array system [8], distillation columns in the chemical industry [9] and semiconductor exposure apparatus [10]. In addition to its numerical simplicity and requiring no iterative procedures, the subspace method is also convenient for optimal estimation and control. However, without special treatment, the subspace method usually gives bias when implemented to a system that works under closed-loop operation. Ljung [1] identified that the problem is due to correlation between process noise (from feedback mechanism) to the input system. Nevertheless, it is desirable to have a subspace approach that works satisfactorily regardless of whether the data is collected in open-loop or closed-loop manner.

The subspace method for continuous time system identification presented in this article is partially influenced by the ideas of Yang [11], with time domain perception instead of frequency domain. Haverkamp [12] gives a similar perspective. Here, the Laguerre filter constructing the $w$-operator is implemented similar to Yang [11] and an instrumental variable adopting to the 
constructing model is similar to Haverkamp's [12]. The instrumental variables are constructed based on the responses of higher order Laguerre filters which reduces the effects of noise and disturbances on the estimate of the state space model. Furthermore, the modified Gram-Schmidt factorisation has provided us with better conditioning in comparison to standard $L U$ factorisation.

The remainder of the article goes as follows. Section 2 presents the algorithm for continuous time system identification using subspace method. The construction of the filtered data matrices is addressed and the framework of identification algorithm is also outlined in Section 2. Section 3 shows the experimental identification results to illustrate the performance of the subspace method on identifying two continuous-time systems: one is a simulated plant with coloured noise while the other is a magnetic bearing system apparatus. Section 4 concludes.

\section{Continuous time system identification}

In mathematical formulation, the continuous time system is given by the state space model equations

$$
\begin{aligned}
\dot{x}(t) & =A x(t)+B u(t), \\
y(t) & =C x(t)+D u(t) .
\end{aligned}
$$

Here, $x(t) \in \mathbb{R}^{n}$ is the state vector, $u(t) \in \mathbb{R}^{m}$ is the measured input signals, and $y(t) \in \mathbb{R}^{l}$ is the measured output signals. Matrices $A \in \mathbb{R}^{n \times n}, B \in$ $\mathbb{R}^{n \times m}, C \in \mathbb{R}^{l \times n}$ and $D \in \mathbb{R}^{l \times m}$ are the system matrices. The $\dot{x}$ denotes the time derivative of $x$.

Next, we introduce the Laguerre filter used in the continuous time identification algorithm. The Laguerre filters are closely related to the first order 
all-pass filter. They also have certain similarities with the discrete time domain $z$-operator [12]. These properties are exploited in the identification of continuous time systems. The $i$ th continuous time Laguerre filter is

$$
L_{i}(s)=\sqrt{2 p} \frac{(s-p)^{i-1}}{(s+p)^{i+1}},
$$

where $p>0$ is the scaling factor to ensure that the filters are stable.

Let us introduce a $w$-operator that corresponds to the all-pass Laguerre filter:

$$
w(s)=\frac{s-p}{s+p}, \quad s=p \frac{1+w}{1-w}, \quad p>0 .
$$

The transformation of the zeroth Laguerre filter $L_{0}(s)=\sqrt{2 p} /(s+p)$ gives $(1-w) / \sqrt{2 p}$. By repetitively multiplying with $w$, a bank of Laguerre filters is obtained with filter order denotes as $\left(\ell_{0}(t), \ell_{1}(t), \ldots, \ell_{i}(t)\right)$. Therefore, the model description in (1-2) is transformed into

$$
\begin{aligned}
& {[w \dot{x}](t)=A_{w} x(t)+B_{w}\left[\ell_{0} u\right](t),} \\
& {\left[\ell_{0} y\right](t)=C_{w} x(t)+D_{w}\left[\ell_{0} u\right](t),}
\end{aligned}
$$

with $\quad A_{w}=\left(A+p I_{n}\right)^{-1}\left(A-p I_{n}\right)$,

$$
\begin{aligned}
& B_{w}=\sqrt{2 p}\left(A+p I_{n}\right)^{-1} B, \\
& C_{w}=\sqrt{2 p} C\left(A+p I_{n}\right)^{-1}, \\
& D_{w}=D-C\left(A+p I_{n}\right)^{-1} B,
\end{aligned}
$$

and

$$
\begin{aligned}
& A=p\left(I_{n}-A_{w}\right)^{-1}\left(I_{n}+A_{w}\right), \\
& B=\sqrt{2 p}\left(I_{n}-A_{w}\right)^{-1} B_{w}, \\
& C=\sqrt{2 p} C_{w}\left(I_{n}-A_{w}\right)^{-1}, \\
& D=D_{w}+C_{w}\left(I_{n}-A_{w}\right)^{-1} B_{w},
\end{aligned}
$$

where $\left[\ell_{i} y\right](t)$ denotes the convolution of $y(t)$ with $\ell_{i}(t)$ and $\left[\ell_{i} y\right](t)=\int_{0}^{t} \ell_{i}(t-$ $\tau) y(\tau) d \tau$ (same implementation to $\left[\ell_{i} u\right](t)$ ). With the transformed system 
description, the continuous time data equation is defined to be

$$
\begin{aligned}
{\left[\begin{array}{c}
{\left[\ell_{0} y\right](t)} \\
{\left[\ell_{1} y\right](t)} \\
\vdots \\
{\left[\ell_{i-1} y\right](t)}
\end{array}\right] } & =\left[\begin{array}{c}
C_{w} \\
C_{w} A_{w} \\
\vdots \\
C_{w} A_{w}^{i-1}
\end{array}\right] x(t) \\
+ & {\left[\begin{array}{cccc}
D_{w} & 0 & \cdots & 0 \\
C_{w} B_{w} & D_{w} & \ddots & \vdots \\
\vdots & \ddots & \ddots & 0 \\
C_{w} A_{w}^{i-2} B_{w} & \cdots & C_{w} B_{w} & D_{w}
\end{array}\right]\left[\begin{array}{c}
{\left[\ell_{0} u\right](t)} \\
{\left[\ell_{1} u\right](t)} \\
\vdots \\
{\left[\ell_{i-1} u\right](t)}
\end{array}\right] }
\end{aligned}
$$

Introduce the notation

$$
\begin{gathered}
Y_{i, j}^{w}(t)=\left[\begin{array}{c}
{\left[\ell_{i} y\right](t)} \\
{\left[\ell_{i+1} y\right](t)} \\
\vdots \\
{\left[\ell_{i+j-1} y\right](t)}
\end{array}\right] ; \quad \Gamma_{j}^{w}=\left[\begin{array}{c}
C_{w} \\
C_{w} A_{w} \\
\vdots \\
C_{w} A_{w}^{j-1}
\end{array}\right] ; \\
H_{j}^{w}=\left[\begin{array}{cccc}
D_{w} & 0 & \cdots & 0 \\
C_{w} B_{w} & D_{w} & \ddots & \vdots \\
\vdots & \ddots & \ddots & 0 \\
C_{w} A_{w}^{j-2} B_{w} & \cdots & C_{w} B_{w} & D_{w}
\end{array}\right]
\end{gathered}
$$

$U_{i, j}^{w}(t)$ is defined similar to $Y_{i, j}^{w}(t)$. With this notation, the continuous time data equation is rewritten in a compact form as

$$
Y_{i, j}^{w}(t)=\Gamma_{j}^{w}\left[w_{i} x\right](t)+H_{j}^{w} U_{i, j}^{w}(t) .
$$

Using the sampled data at sampling times $t_{1}, t_{2}, \ldots, t_{N}$, the sampled data matrices are

$$
Y_{i, j, N}^{w}=\left[\begin{array}{cccc}
{\left[\ell_{i} y\right]\left(t_{1}\right)} & {\left[\ell_{i} y\right]\left(t_{2}\right)} & \cdots & {\left[\ell_{i} y\right]\left(t_{N}\right)} \\
{\left[\ell_{i+1} y\right]\left(t_{1}\right)} & {\left[\ell_{i+1} y\right]\left(t_{2}\right)} & \cdots & {\left[\ell_{i+1} y\right]\left(t_{N}\right)} \\
\vdots & \vdots & \ddots & \vdots \\
{\left[\ell_{i+j-1} y\right]\left(t_{1}\right)} & {\left[\ell_{i+j-1} y\right]\left(t_{2}\right)} & \cdots & {\left[\ell_{i+j-1} y\right]\left(t_{N}\right)}
\end{array}\right]
$$




$$
X_{i, N}^{w}=\left[\begin{array}{llll}
{\left[w_{i} x\right]\left(t_{1}\right)} & {\left[w_{i} x\right]\left(t_{2}\right)} & \cdots & {\left[w_{i} x\right]\left(t_{N}\right)}
\end{array}\right] .
$$

With these matrices, the sampled data equation becomes

$$
Y_{i, j, N}^{w}(t)=\Gamma_{j}^{w} X_{i, N}^{w}(t)+H_{j}^{w} U_{i, j, N}^{w}(t) .
$$

Now, the output data sequences is divided into two categories known as past and future output. Past output is denoted by constructing the data matrices from 0 th to $(i-1)$ th order and is represented by $Y_{0, i, N}^{w}$, whereas the future output is denoted by constructing the data matrices from $i$ th to $(j-1)$ th order and is represented by $Y_{i, j, N}^{w}$. Similar construction of data matrices is applied to past and future input, and is represented as $U_{0, i, N}^{w}$ and $U_{i, j, N}^{w}$ respectively.

Next, we introduce the projection on the null space of $U_{0, i, N}^{w}$,

$$
\Pi_{U_{0, i, N}}^{\perp}=I-U_{0, i, N}^{\top}\left(U_{0, i, N} U_{0, i, N}^{\top}\right)^{-1} U_{0, i, N} .
$$

By multiplying (14) to both side of (13) the term $H_{j}^{w} U_{0, i, N}^{w}$ will be removed as $U_{0, i, N} \Pi_{0, i, N}^{\perp}=0$. Therefore, we obtain

$$
Y_{0, i, N}^{w} \Pi_{U_{0, i, N}}^{\perp}=\Gamma_{i}^{w} X_{0, N}^{w} \Pi_{U_{0, i, N}}^{\perp} .
$$

Equation (15) produces a state space model with reasonable quality if the noise level in the system is sufficiently small. However, for most of the system the noise existence either from process or measurement noise is often unavoidable. To reduce the effect of noise, we propose to use instrumental variables in the identification of state space models.

We construct the instrumental variables using future input and future output data, where the instrumental data matrix

$$
Z=\left[\begin{array}{c}
U_{i, j, N}^{w} \\
Y_{i, j, N}^{w}
\end{array}\right]
$$

Now multiply again (15) with projection matrix of $\Pi_{Z}^{\perp}$ to obtain

$$
\lim _{N \rightarrow \infty} \frac{1}{N} Y_{0, i, N} \Pi_{U_{0, i, N}}^{\perp} \Pi_{Z}^{\perp}=\lim _{N \rightarrow \infty} \frac{1}{N} \Gamma_{j} X_{i, N} \Pi_{U_{0, i, N}}^{\perp} \Pi_{Z}^{\perp} .
$$


Another matter to be considered is the effect of initial state condition of the state space system. This problem is overcome by introducing data matrices constructed from the set of Laguerre filter. Here,

$$
\Psi_{i, j, N}=\left[\begin{array}{cccc}
\ell_{i}\left(t_{1}\right) & \ell i\left(t_{2}\right) & \cdots & \ell_{i}\left(t_{N}\right) \\
\ell_{i+1}\left(t_{1}\right) & \ell_{i+1}\left(t_{2}\right) & \cdots & \ell_{i+1}\left(t_{N}\right) \\
\vdots & \vdots & \ddots & \vdots \\
\ell_{i+j-1}\left(t_{1}\right) & \ell_{i+j-1}\left(t_{2}\right) & \cdots & \ell_{i+j-1}\left(t_{N}\right)
\end{array}\right]
$$

The past Laguerre filter bank is used for causal case and is denoted by $\Psi_{0, i, N}$ while the future Laguerre filter bank is used for anti-causal case and is denoted by $\Psi_{i, j, N}$. This term results in the exponential decay of the initial state of the state space system.

\subsection{Constructing filtered data matrices}

There are few ways that could be implemented in order to generate the Laguerre functions [13]. Here we use the numerical solution of the differential equations

$$
\left[\begin{array}{c}
i_{1}(t) \\
\dot{l}_{2}(t) \\
\vdots \\
i_{i}(t)
\end{array}\right]=\left[\begin{array}{cccc}
-p & 0 & \cdots & 0 \\
-2 p & -p & \cdots & 0 \\
\vdots & \ddots & \ddots & \vdots \\
-2 p & \cdots & -2 p & -p
\end{array}\right]\left[\begin{array}{c}
l_{1}(t) \\
l_{2}(t) \\
\vdots \\
l_{i}(t)
\end{array}\right]
$$

with the initial conditions

$$
\left[\begin{array}{c}
l_{1}(0) \\
l_{2}(0) \\
\vdots \\
l_{i}(0)
\end{array}\right]=\sqrt{2 p}\left[\begin{array}{c}
1 \\
1 \\
\vdots \\
1
\end{array}\right]
$$


Hence, a set of continuous time Laguerre functions are found numerically by iteratively solving the difference equations

$$
\left[\begin{array}{c}
l_{1}\left(t_{a+1}\right) \\
l_{2}\left(t_{a+1}\right) \\
\vdots \\
l_{i}\left(t_{a+1}\right)
\end{array}\right] \approx\left[\begin{array}{cccc}
-p & 0 & \cdots & 0 \\
-2 p & -p & \cdots & 0 \\
\vdots & \ddots & \ddots & \vdots \\
-2 p & \cdots & -2 p & -p
\end{array}\right]\left[\begin{array}{c}
l_{1}\left(t_{a}\right) \\
l_{2}\left(t_{a}\right) \\
\vdots \\
l_{i}\left(t_{a}\right)
\end{array}\right] \times \Delta t+\left[\begin{array}{c}
l_{1}\left(t_{a}\right) \\
l_{2}\left(t_{a}\right) \\
\vdots \\
l_{i}\left(t_{a}\right)
\end{array}\right]
$$

with

$$
\left[\begin{array}{c}
l_{1}\left(t_{0}\right) \\
l_{2}\left(t_{0}\right) \\
\vdots \\
l_{i}\left(t_{0}\right)
\end{array}\right]=\sqrt{2 p}\left[\begin{array}{c}
1 \\
1 \\
\vdots \\
1
\end{array}\right]
$$

and $\Delta t=t_{a+1}-t_{a}$ being the integration step size (sampling rate).

To generate the filtered input and output, and instead of performing a convolution, the data matrices are developed via implementation of the solution of the differential equation

$$
\left[\begin{array}{c}
\dot{z}_{1}(t) \\
\dot{z}_{2}(t) \\
\vdots \\
\dot{z}_{i}(t)
\end{array}\right]=\left[\begin{array}{cccc}
-p & 0 & \cdots & 0 \\
-2 p & -p & \cdots & 0 \\
\vdots & \ddots & \ddots & \vdots \\
-2 p & \cdots & -2 p & -p
\end{array}\right]\left[\begin{array}{c}
z_{1}(t) \\
z_{2}(t) \\
\vdots \\
z_{i}(t)
\end{array}\right]+\sqrt{2 p}\left[\begin{array}{c}
1 \\
1 \\
\vdots \\
1
\end{array}\right] y(t)
$$

Therefore, a set of filtered output is generated numerically by iteratively solving the difference equations

$$
\left[\begin{array}{c}
y_{1}^{f}(t) \\
y_{2}^{f}(t) \\
\vdots \\
y_{i}^{f}(t)
\end{array}\right] \approx\left[\begin{array}{c}
y_{1}^{f}(t) \\
y_{2}^{f}(t) \\
\vdots \\
y_{i}^{f}(t)
\end{array}\right]+\left[\begin{array}{cccc}
-p & 0 & \cdots & 0 \\
-2 p & -p & \cdots & 0 \\
\vdots & \ddots & \ddots & \vdots \\
-2 p & \cdots & -2 p & -p
\end{array}\right]\left[\begin{array}{c}
y_{1}^{f}(t) \\
y_{2}^{f}(t) \\
\vdots \\
y_{i}^{f}(t)
\end{array}\right] \times \Delta t
$$




$$
+\sqrt{2 p}\left[\begin{array}{c}
1 \\
1 \\
\vdots \\
1
\end{array}\right] y(t) \times \Delta t
$$

with zero initial condition of $y_{i}^{f}(t)$. The filtered input is also formed in a similar way.

\subsection{Identification using a causal IV}

Let $L_{i}(s)$ be a bank of causal Laguerre filters $(p>0)$. Let $u(t)$ and $y(t)$ be the input and output plant data described in (1) and (2). Let $U_{0, i, N}^{w}$, $Y_{0, i, N}^{w}, U_{i, j, N}^{w}$ and $Y_{i, j, N}^{w}$ be constructed from $u(t)$ and $y(t)$, according to (11) and $\Psi_{0, i, N}$ as in (18).

Consider the $R Q$ factorisation

$$
\left[\begin{array}{c}
\Psi_{0, i, N} \\
U_{0, i, N}^{w} \\
U_{i, j, N}^{w} \\
Y_{i, j, N}^{w} \\
Y_{0, i, N}^{w}
\end{array}\right]=\left[\begin{array}{ccccc}
R_{11} & 0 & 0 & 0 & 0 \\
R_{21} & R_{22} & 0 & 0 & 0 \\
R_{31} & R_{32} & R_{33} & 0 & 0 \\
R_{41} & R_{42} & R_{43} & R_{44} & 0 \\
R_{51} & R_{52} & R_{53} & R_{54} & R_{55}
\end{array}\right]\left[\begin{array}{c}
Q_{1} \\
Q_{2} \\
Q_{3} \\
Q_{4} \\
Q_{5}
\end{array}\right] .
$$

Then

$$
\lim _{N \rightarrow \infty} \frac{1}{\sqrt{N}}\left[\begin{array}{ll}
R_{53} & R_{54}
\end{array}\right]=\lim _{N \rightarrow \infty} \frac{1}{\sqrt{N}} \Gamma_{i}^{w} X_{0, N}^{w}\left[\begin{array}{l}
Q_{3} \\
Q_{4}
\end{array}\right]^{\top}
$$

Proof: From the the $R Q$ factorisation of (25) we have

$$
\lim _{N \rightarrow \infty} \frac{1}{\sqrt{N}}\left[\begin{array}{ll}
R_{53} & R_{54}
\end{array}\right]=\lim _{N \rightarrow \infty} \frac{1}{\sqrt{N}} Y_{0, i, N}^{w}\left[\begin{array}{l}
Q_{3} \\
Q_{4}
\end{array}\right]^{\top} .
$$


From (13) and (17)

$$
\lim _{N \rightarrow \infty} \frac{1}{\sqrt{N}} Y_{0, i, N}^{w}\left[\begin{array}{l}
Q_{3} \\
Q_{4}
\end{array}\right]^{\top}=\lim _{N \rightarrow \infty} \frac{1}{\sqrt{N}} \Gamma_{i}^{w} X_{0, N}^{w}\left[\begin{array}{l}
Q_{3} \\
Q_{4}
\end{array}\right]^{\top}
$$

The terms due to the input and the initial state are zero because of the orthogonality between $\left(Q_{1}, Q_{2}\right)$ and $\left(Q_{3}, Q_{4}\right)$. The noise terms will also disappear as $N$ goes to infinity.

\subsection{Identification algorithm}

The identification procedure used is based on the causal Laguerre filter. The subspace algorithm to identify the continuous time system is the following.

1. Construct the filtered data matrices of $U_{0, i, N}^{w}, U_{i, j, N}^{w}, Y_{0, i, N}^{w}$ and $Y_{i, j, N}^{w}$ according to (24), and $\Psi_{0, i, N}$ according to (21).

2. Perform the $R Q$ decomposition

$$
\left[\begin{array}{c}
\Psi_{0, i, N} \\
U_{0, i, N}^{w} \\
U_{i, j, N}^{w} \\
Y_{i, j, N}^{w} \\
Y_{0, i, N}^{w}
\end{array}\right]=\left[\begin{array}{ccccc}
R_{11} & 0 & 0 & 0 & 0 \\
R_{21} & R_{22} & 0 & 0 & 0 \\
R_{31} & R_{32} & R_{33} & 0 & 0 \\
R_{41} & R_{42} & R_{43} & R_{44} & 0 \\
R_{51} & R_{52} & R_{53} & R_{54} & R_{55}
\end{array}\right]\left[\begin{array}{c}
Q_{1} \\
Q_{2} \\
Q_{3} \\
Q_{4} \\
Q_{5}
\end{array}\right] .
$$

3. Perform the singular value decomposition (SVD) to the working matrix $\left[\begin{array}{ll}R_{53} & R_{54}\end{array}\right]$ :

$$
\left[\begin{array}{ll}
R_{53} & R_{54}
\end{array}\right]=U S V^{\top} .
$$

4. Determine the model order $n$ from the singular value in $S$, and construct $U_{n}$ from the first $n$ columns of $U$. Take $U_{1}$ as the upper $(i-$ 1) $l$ rows of $U_{n}$ and $U_{2}$ the lower $(i-1) l$ rows of $U_{n}$. 
5. Compute $A_{w}$ and $C_{w}$ :

$$
\begin{aligned}
& C_{w}=\text { the upper } l \text { rows of } U_{n}, \\
& A_{w}=U_{1}^{\dagger} U_{2} .
\end{aligned}
$$

6. The $A$ and $C$ are obtained using the relations

$$
\begin{aligned}
& A=p\left(I_{n}+A_{w}\right)\left(I_{n}-A_{w}\right)^{-1}, \\
& C=\sqrt{2 p} C_{w}\left(I_{n}-A_{w}\right)^{-1} .
\end{aligned}
$$

7. Solve least squares problem from model structure

$$
y(t \mid B, D)=C\left(q I_{n}-A\right)^{-1} B u(t)+D u(t) .
$$

8. Reconstruct $B$ and $D$ from $(B, D)$.

9. Generate the predicted output.

\section{Simulation examples}

To demonstrate the performance of the proposed approach in identifying a continuous time system, we chose two sets of data: a simulated system with coloured noise disturbance (System 3.1); and a set of real plant data from magnetic bearing system apparatus (System 3.2).

\subsection{Coloured noise}

System 3.1 has the transfer function

$$
G(s)=\frac{100}{(s+1)(s+3)} .
$$



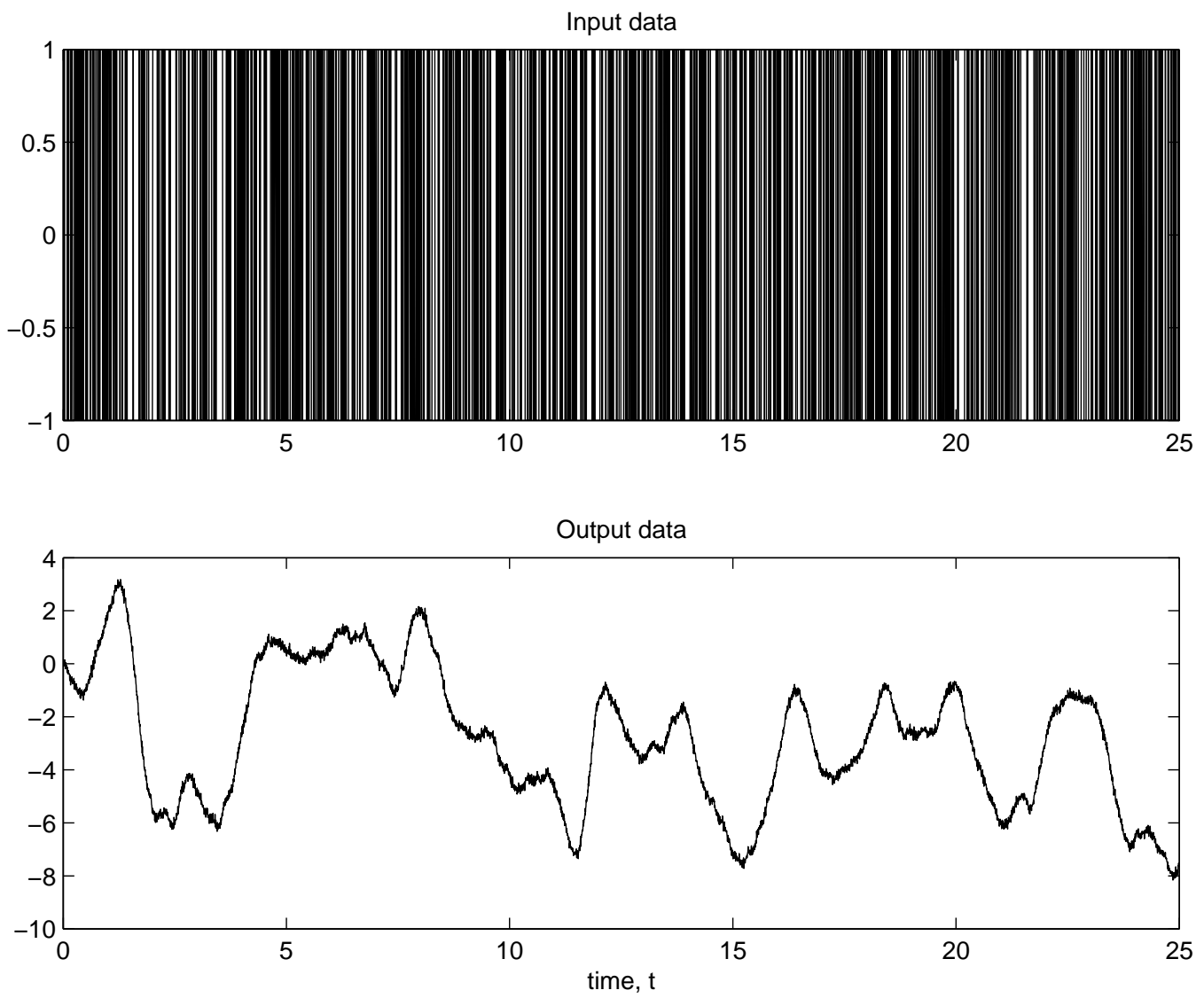

Figure 1: Input and output data System 3.1 
The input signal, $u(t)$ is generated using a Gaussian random binary signal (GRBS) with a coloured noise sequence. At sampling time, $t=0.005 \mathrm{~s}$, 5000 sets of input and output data is obtained. Then, the output is contaminated with a random walk type disturbance with a discrete filter $V(z)=$ $0.1 /(z-0.1)$. Figure 1 shows the plot of input and output data. This data set is further divided into estimation data set and validation data set. Related design parameters used in the algorithm are set to $i=10$ and $p=1$. The performance of the estimated model is assessed based on the fit between the measured output and the estimated one. The comparison results of estimation and validation data sets with the predicted outputs from the model obtained using subspace method is shown in Figure 2. The result shows that the model could describe the system closely. Further verification tests on mean square error (MSE) and system variance give MSE $=0.0105$ and $\mathrm{VAR}=0.9985$ for estimation data and $\mathrm{MSE}=0.0096$ and $\mathrm{VAR}=0.9976$ for validation data. This shows that the model is able to identify the system with low MSE and good percentage of accuracy even to a validation data set that was not used in the estimation. The Bode plot of system frequency response is also compared with the model's frequency response as in Figure 3.

\subsection{Magnetic bearing system}

The second data set is taken from a magnetic bearing system apparatus. This system has four input and four output system that maintain the position of rotor on $x$-axis and $y$-axis for two-sided, left and right bearing. As for the single input and single output case, four sets of input output data are collected from the experimental apparatus. However, this article only shows one experimental result as the other three can be treated in the same manner. The sampling interval is $\Delta t=7.8125 \times 10^{-4} \mathrm{~s}, 1024$ samples of input and output data are collected. Figure 4 plots the input and output data. Again, the data set is divided into an estimation data set and a validation data set. Related design parameters used in the algorithm are set to $i=10$ and $p=1$. The comparison results of 512 data points of estimation and validation 

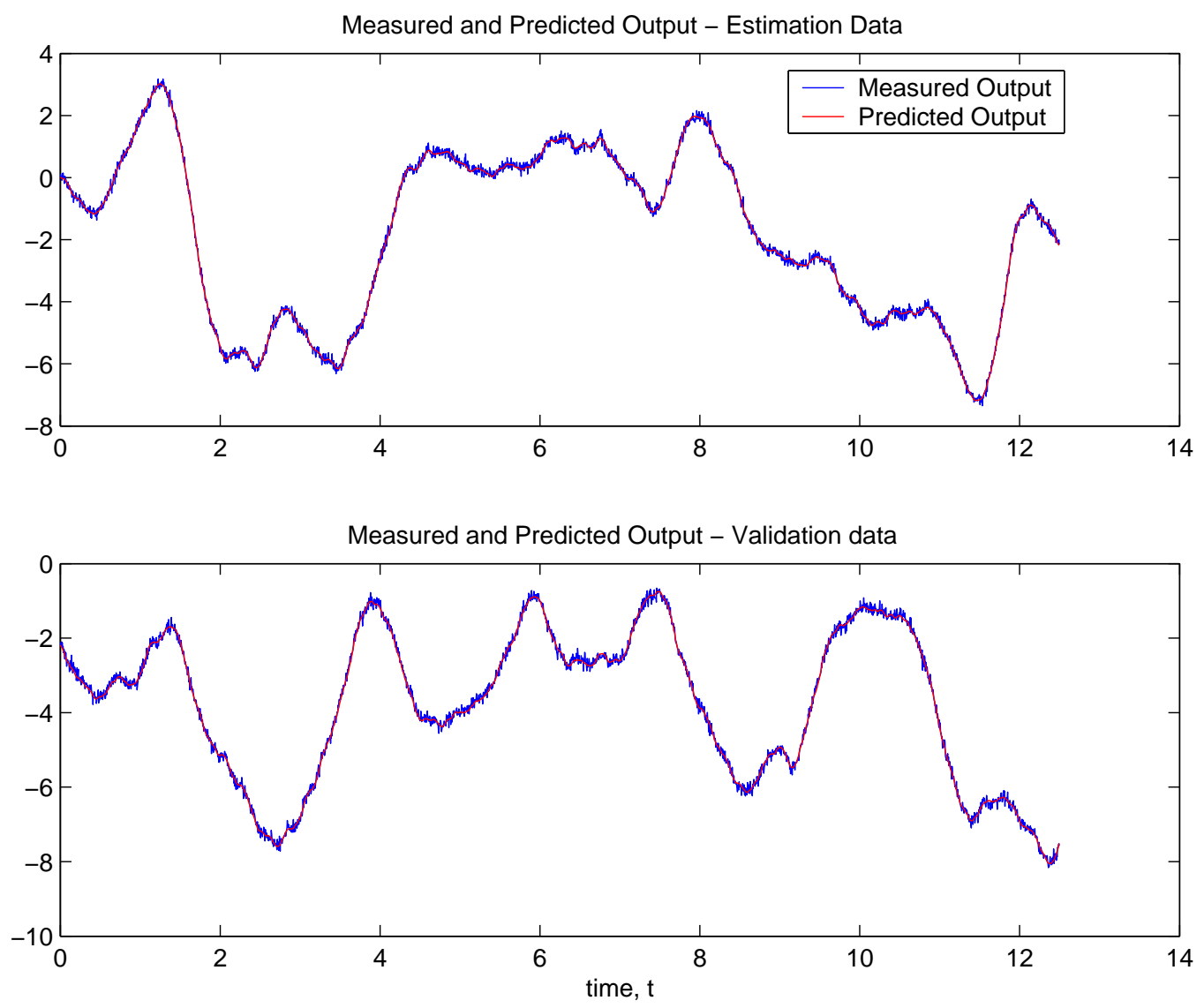

Figure 2: Comparison between predicted output and the system output System 3.1 

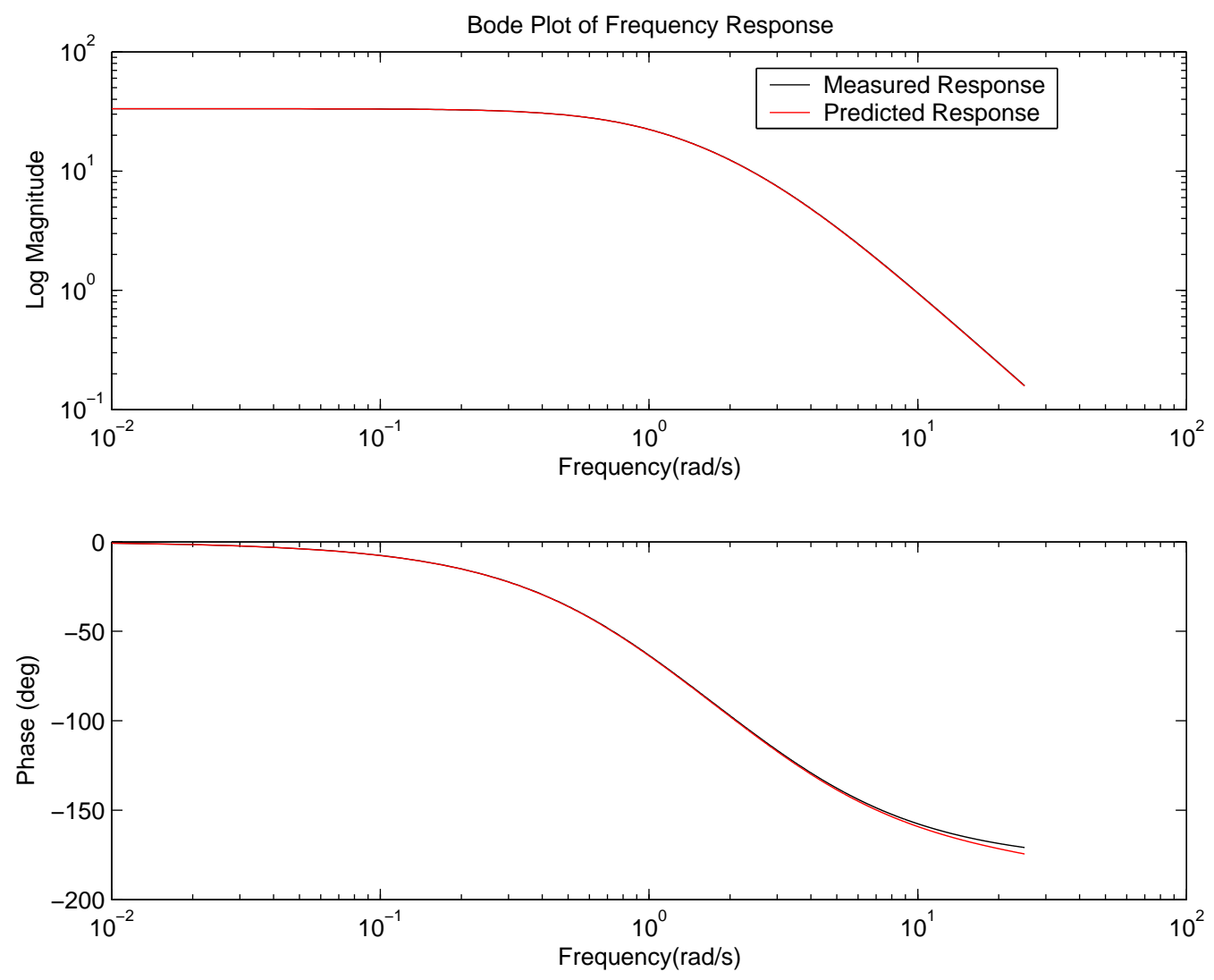

Figure 3: Comparison of model and system frequency response 

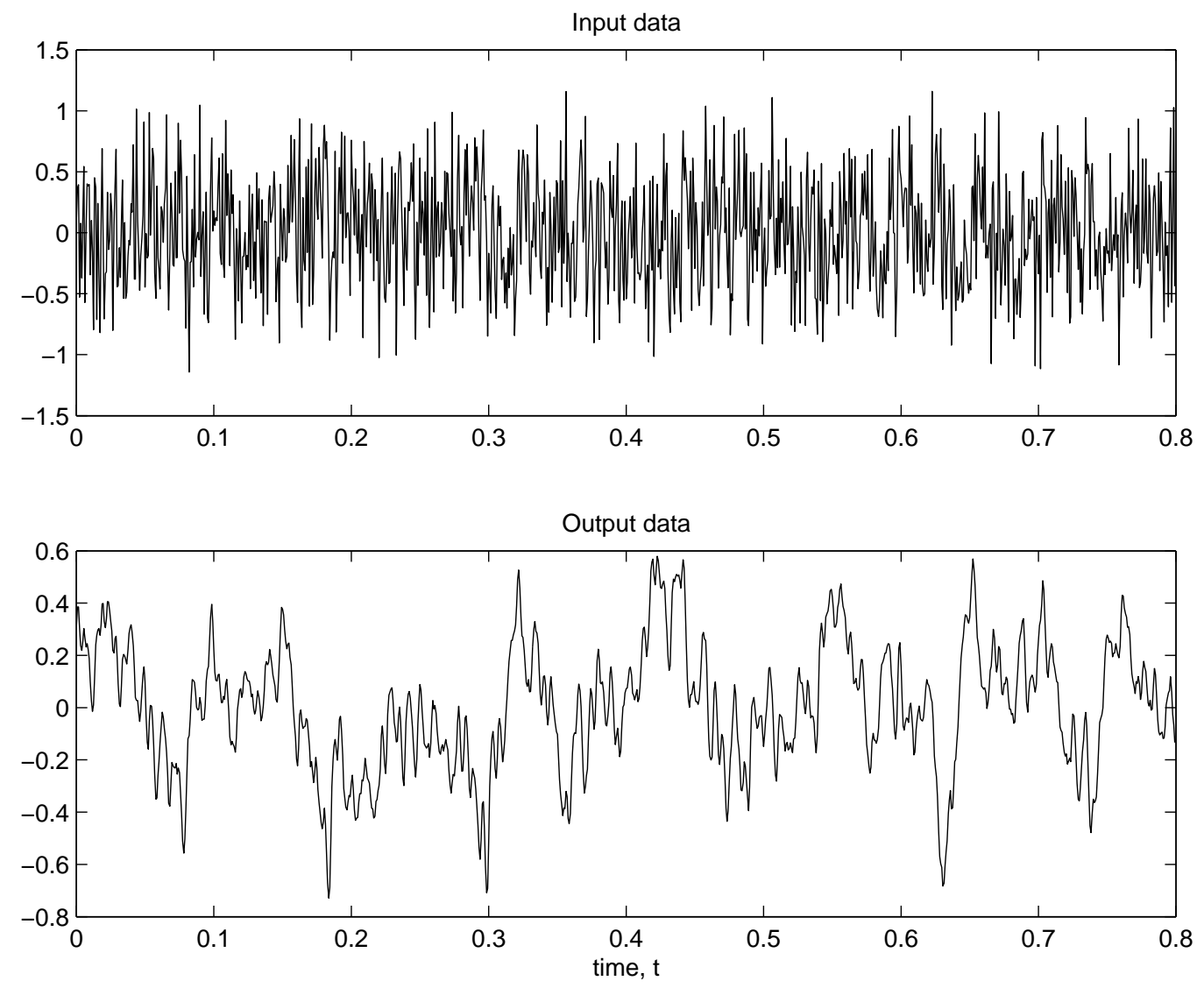

Figure 4: Input and output data of System 3.2 
data sets between the model's output and system's output are shown in Figure 5. Verification tests on mean square error (MSE) and system variance give $\mathrm{MSE}=0.0113$ and $\mathrm{VAR}=0.7830$ for estimation data and MSE $=0.0125$ and $\mathrm{VAR}=0.7936$ for validation data.

\section{Conclusion}

We presented a subspace method to identify a continuous time state space model using instrumental variables. The innovation of constructing filtered data matrices using differential equations provides better computation and easily maintainable parametrisation. In addition, the use of causal Laguerre filters and instrumental variables improves the quality of the model in the presence of measurement noise. This approach was applied to the set of simulated data and the set of experimental data generated from a magnetic bearing system apparatus. Both applications show the efficacy of the proposed algorithm.

\section{References}

[1] L. Ljung, System Identification: Theory for the User, Prentice Hall, New Jersey, 1999. C713, C714

[2] G. C. Goodwin, S. T. Graebe and M. E. Salgado, Control System Design, Prentice Hall, New Jersey, 2001. C713

[3] T. McKelvey, H. Akcay and L. Ljung, Subspace-based Multivariable System Identification from Frequency Response Data, IEEE Trans. on Automatic Control, 41(7), 960-979, 1996. C714 

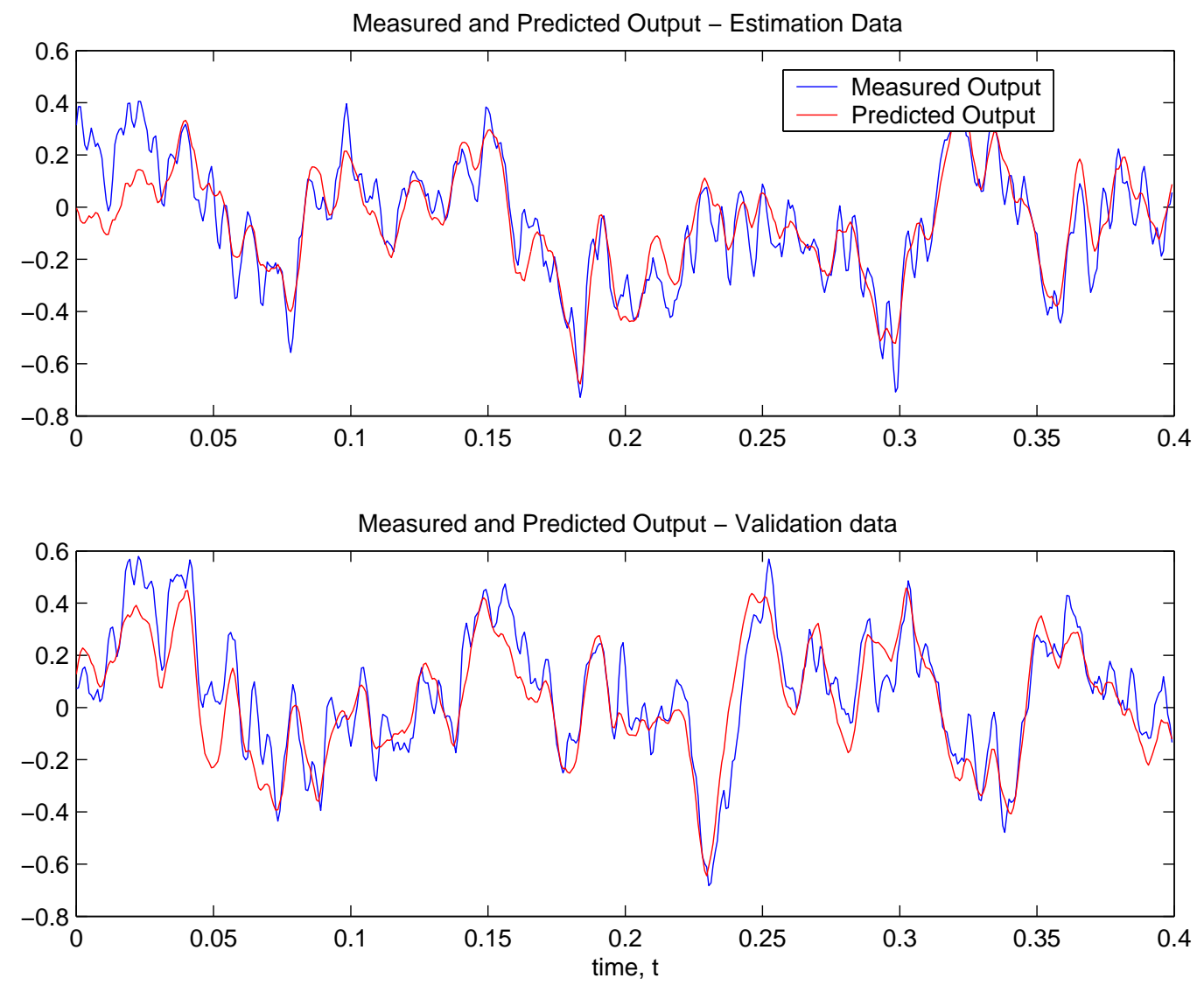

Figure 5: Comparison between the predicted output and the system's output of System 3.2 
[4] T. Gustafsson, M. Lovera and M. H. Verhaegen, A Novel Algorithm for Recursive Instrumental Variable Based Subspace Identification, in Proc. of IEEE Conf. on Decision and Control, 3920-3925, 1998. C714

[5] F. Demourant and G. Ferreres, A Frequency Domain Identification-control Approach for A Flexible Aircraft, in Proc. of IEEE Int. Conf. on Control Applications, 126-137, 2002. C714

[6] B. R. J. Haverkamp, C. T. Chou, M. H. Verhaegen and R. Johansson, Identification of continuous-time MIMO state space models from sampled data in the presence of process and measurement noise, in Proc. of 35th. Conf. on Decision and Control, 1539-1544, 1996. C714

[7] H. Akcay, S. M. Islam and B. Ninnes, Subspace-based identification of power transformer models from frequency response data, IEEE Trans. on Inst. and Measurement, 48(3), 700-704, 1999. C714

[8] H. L. Ari and A. J. Devancy, The time-reversal technique re-interpreted: Subspace-based signal processing for multi-static target location, in Proc. of IEEE on Sensor Array and Multi-channel Signal Processing Workshop, 509-513, 2000. C714

[9] W. Favoreel, B. De Moor and P. Van Overschee, Subspace identification of bilinear systems subject to white inputs, IEEE Trans. on Automatic Control), 44(6), 1157-1165, 1999. C714

[10] H. Kato, S. Wakui, T. Mayama, A. Toukairin, H. Takanashi and S. Adashi, System identification of anti-vibration units in semiconductor exposure apparatus, in Proc. of IEEE Int. Conf. on Control Applications, 1312-1317, 1999. C714

[11] Z.J. Yang, Frequency domain subspace model identification with the aid of the w-operator, in Proc. of the SICE Annual Conf., 1077-1080, 1998. C714 
[12] B. J. R. Haverkamp, Subspace Method: Identification, Theory and Practice, Ph.D. Thesis, Faculty of Information Technology and Systems, Delft University, The Netherlands, 2000. C714, C715, C716

[13] L. Wang and W. R. Cluett, From Plant Data to Process Control: Ideas for Process Identification and PID Design, Francis \& Taylor, London, 2000. C719 